\title{
Fluoride varnish reduces white spot lesions during orthodontic treatment
}

\section{Do regular fluoride applications reduce the development of white spot lesions when people are undergoing fixed orthodontic treatment?}

\author{
Stecksén-Blicks C, Renfors G, Oscarson ND, Bergstrand F, \\ Twetman S. \\ Caries-preventive effectiveness of a fluoride varnish: a randomized \\ controlled trial in adolescents with fixed orthodontic appliances. \\ Caries Res 2007; 41:455-459
}

Design This was a randomised controlled trial (RCT) set in a community dental practice.

Intervention The test varnish was a commercially available product, Fluor Protector (Ivoclar Vivadent, Schaan, Liechtenstein), containing $0.1 \%$ fluoride as difluorosilane in a polyurethane varnish base. The placebo varnish applied had an identical composition but without fluoride.

Outcome measure The incidence and prevalence of white spot lesions (WSL) on the upper incisors, cuspids and premolars were recorded, as scored from digital photographs by two independent examiners. In the case of disagreement, cases were re-examined until a consensus was achieved.

Results The incidence of WSL during the treatment period was $7.4 \%$ in the fluoride varnish group compared with $25.3 \%$ placebo group $(P<0.001)$. The mean progression score was significantly lower in the fluoride varnish group than in the placebo group, $(0.8 \pm 2.0$ vs $2.6 \pm 2.8$; $\mathrm{P}<0.001)$. The absolute risk reduction was $18 \%$ and the number-neededto-treat was calculated to be 5.5 (95\% confidence interval, 3.7-10.9).

Conclusions The results strongly suggest that regular topical fluoride varnish applications may reduce the development of WSL adjacent to the bracket base during treatment with fixed appliances.

\section{Commentary}

Fixed appliance orthodontic therapy can be considered a risk factor in the formation of the aesthetically unattractive WSL. Preventing the accumulation of dental plaque around the surface of appliance components requires high standards of oral hygiene. Preventative measures advised during orthodontic therapy include the slurry toothbrushing effect, ${ }^{1}$ when the patient spits out their fluoridated toothpaste at the conclusion of toothbrushing, instead of rinsing with water. A daily rinse with $0.05 \%$ sodium fluoride mouthwash for fixed appliance therapy patients is also recommended, ${ }^{2}$ but these methods require a high level of compliance on the part of the patient. The research described here provides evidence of effectiveness of an alternative preventive measure that is not so reliant on patient co-operation.

This double-blind RCT aimed to evaluate the efficiency of topical fluoride varnish applications on the prevention of WSL formation in adolescents during fixed orthodontic appliance therapy. The study was generally well designed, although the randomisation allocation could have been open to bias since it involved the roll of a dice. The use of a random number generator or third party randomisation would have been more robust. Baseline data collected for both groups described caries prevalence (decayed, missing, filled surfaces), availability of piped drinking water and age. Inclusion of other variables, such as gender, socio economic status and oral hygiene habits, would have provided useful additional information on known confounding factors.

Clinical digital photographs were utilised as the method for recording and evaluating maxillary anterior teeth for the presence of WSL. Photographs can be retained and examined later, but are not without problems although Kappa scores for inter- and intra-examiner reliability tests showed substantial levels of agreement of 0.69 and 0.77 respectively.

The results provide an overview of the patient flow in a CONSORT $^{3}$ flow diagram, which describes the participants in the trial with respect to inclusions, dropouts and numbers. However, the diagram omits those initially selected in the sample who did not consent to participate in the trial. The number of dropouts is small, so an intent-to-treat analysis would have had little impact on the results, which show a significant $18 \%$ reduction in the risk of WSL in the varnish group.

In summary, the paper was lucidly written and reasonable conclusions were drawn. The application of this technique in dental practice could result in greater clinical control of these patients' therapy, since the advice to use a daily mouthrinse may have variable compliance. The trial provides good evidence that fluoride varnish application can reduce the development of WSL adjacent to brackets bases during fixed appliance treatment.

\section{Imran Shafi}

Glasgow Dental Hospital and School, Glasgow, Scotland, UK

1. Sjogren $\mathrm{K}$, Ekstrand J, Birkhed D. Effect of water rinsing after toothbrushing on fluoride ingestion and absorption. Caries Res 1994; 28:455-459.

2. Benson PE, Parkin N, Millett DT, Dyer FE, Vine S, Shah A. Fluorides for the prevention of white spots on teeth during fixed brace treatment. Cochrane Database Syst Rev 2004; issue 3.

3. Altman DG, Schulz KF, Moher D, et al. The revised CONSORT statement for reporting randomized trials: explanation and elaboration. Ann Int Med 2001; 134:663-694.

Evidence-Based Dentistry (2008) 9, 81. doi:10.1038/sj.ebd.6400599 